\title{
Analysis Method of Dynamic Extensible Service Model Based On Petri-Net
}

\author{
Zhang Can $^{1 a}$, Jiang Jing*, Fan Cheng, Sun Jie, Yang Rui-Min \\ 1College of Information Engineering, QingDao University, QingDao,ShanDong, 266071, China \\ ${ }^{*}$ College of Information Engineering, QingDao University, QingDao,ShanDong, 266071, China \\ a zhangcan_12345678@163.com \\ *jj@qdu.edu.cn
}

Key words: Petri net, on-demand services, application customization, dynamic extensible

\begin{abstract}
It is a big challenge to realize the dynamic change of the network environment and user's demands. A dynamic application customization and extensible service composition model is proposed in this paper. In order to guarantee the rationality of the service composition, the composition model of on-demand service that based on Petri net is proposed. Then the model is analyzed to validate whether the service composition can achieve the desired target. Finally, the result shows that the model can guarantee the correctness of extensible service, validate the rationality and effectiveness of the method.
\end{abstract}

\section{Introduction}

In the emerging paradigm of Service-oriented computing, data, software and hardware can all be encapsulate as service shared on the Internet. Application would no longer be built from scratch, but as compositions of the available services. In this way, resources can be integrated, which are dynamic, distributed, multi-domain and within the virtual organization resources, to achieve the purpose of solving collaborative problem ${ }^{[1,2]}$.

With the development of Service-oriented computing technology, the number of published services gets larger and larger. The services are selected and composed into application customization to fulfill user's personal demands. On the other hand, in order to fulfill user's dynamic demands, how to describe user's requirements exactly and dynamic select the suitable services to extend are the most challenging problems in the field of on-demand service.

User's requirements are often at a high level of abstraction and very personalized; on the other hand, at the purpose of reusing, Web services are all designed to meet specific demands. In order to fulfill user's dynamic demands, it should be designed that the composition model has an application function and dynamic extensible service. In this model user's personal requirements can be described exactly and composed suitable services into application.

Web service composition language, such as WSFL, WSCI, and BPML, just describe the process, which some basic Web services composed into a complex service; can not verify the correctness of services. But Petri net can model the complex system and verify the correctness of the model .Therefore, the modeling method of on-demand application service based on Petri net is studied in this paper. And the on-demand application service is formally modeled by Petri net. For dynamic extensible system can run on the network and provide continuous services, dynamic extensible service composition model based on Petri net is proposed, then analyzes the reachability, boundedness, liveness, integrity and progressivity of the model, to validate the service.

The remainder of this paper is organized as follows. Section 2 shows formal definition of on-demand application customization service model and its service model. Section 3 shows the basis how to analysis the correctness of model, constructs the reachability tree of dynamic extensible model, and verifies whether the model is correctness and rationality. Section 4 summarizes the article and the future research direction in the future. 


\section{On-demand Service Model Description}

Petri net is a kind of tool that is graphic, mathematics modeling tool and suitable for multisystem, provides a powerful means to described and studied with complex structure systems which have parallel, asynchronous, distributed and randomness features ${ }^{[3]}$.

Petri net has five basic elements: place, transition, token, connection, the initial marking $\mathrm{M}_{0}$. In Petri net, place is represented by the round node; transition is represented by the square node; connection is a place and transition between the directed arc; token is a dynamic objects, which can be moved from one place to another place. The result of transition is that remove a token from the input place to the output place.

\section{Formal Definition of On-demand Application Customization Service}

An on-demand application customization service instance can be mapped into a Petri net. The operation of services maps the transition of Petri net, and the state of services maps the place of Petri net, and the relationship between the operation and the status is mapped into connection between transition and place. On-demand application service based on Petri net is defined as follows:

Definition 1. A on-demand application customization service can be expressed as a 6-tuple: On-demand Application Customization Service S= (OASID, OASN, OASD, OASS, EF, SN)

OASID—on-demand application service unique identification number

OASN_-on-demand application service’s name

OASD - on-demand applications service description, such as the description of service call address;

OASS - the service or operation provided by on-demand application service

$\mathrm{EF}$ - the extensible flag of on-demand service, when EF = true indicates that the service is extended services, or the service is not extended services

$\mathrm{SN}=\left(\mathrm{P}, \mathrm{T}, \mathrm{W}, \mathrm{i}, \mathrm{o}, \mathrm{M}_{0}\right)$, it is the on-demand application customization service corresponding Petri net (Service Net).

$\cdot \mathrm{P}$ is a finite place set, where each library represents a state. $\mathrm{P}=\left\{\mathrm{p}_{1}, \mathrm{p}_{2}, \ldots ., \mathrm{p}_{\mathrm{n}}\right\}$;

- $\mathrm{T}$ is a finite set of transition, represents service operation and the operation between services. $\mathrm{T}=\left\{\mathrm{t}_{1}, \mathrm{t}_{2}, \ldots . ., \mathrm{t}_{\mathrm{n}}\right\}$;

- $\mathrm{W} \subseteq(\mathrm{P} \times \mathrm{T}) \mathrm{U}(\mathrm{T} \times \mathrm{P})$ is a collection of connection, in which each connection represents the causal relationship from the operate to state or from the state to operate;

$\cdot \mathrm{i}$ is the input place. $\mathrm{i}=\varnothing$; $\mathrm{i}$ is the initial state of the service $\mathrm{S}$. only when place $\mathrm{i}$ has a token, the service $\mathrm{S}$ can be performed.

$\cdot 0$ is the output place. $o=\varnothing$; When there is a token in the place $o$, then service $S$ is finished.

$\cdot \mathrm{M}_{0}$ is the initial marking of $\mathrm{SN}$, and has the initial state of all place form SN.

\section{On-demand Application Service Based on Petri Net Modeling}

Any time a service instance can be at the following several states: not instantiated, ready, running, suspended, finished and fail.

What's more, if definition of service model is rationality, it must meet the following criteria:

(a) Each model has only one input place i and output place 0.

(b) The service is completed, if and only if the output place o has a token.

(c) All the transitions and places are all in the path from the input place i to the output place o.

The basic models which are mainly applied on on-demand application service model are sequence model, choice model and call model.

a. Sequence

Sequence (S1, S2) shows that the combination service is formed, after the implementation sequentially of S1 andS2, as shown in Fig. 1.

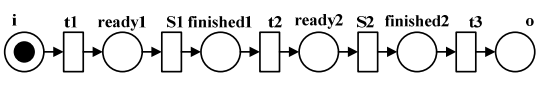

Fig. 1 Sequence (S1, S2)

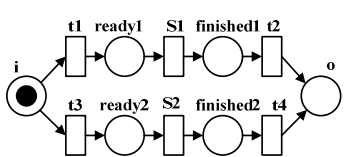

Fig. 2 Choice (S1, S2) 


\section{b. Choice}

Choice (S1, S2) represents that a combination of the service is formed, after the implementation of S1 or S2 (non-concurrently), once one of them is executed, the other one is not performed, as shown in Fig. 2

c. Call

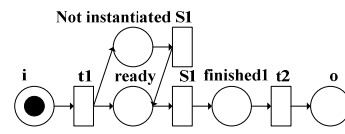

Fig. 3a Call (S1, S1)

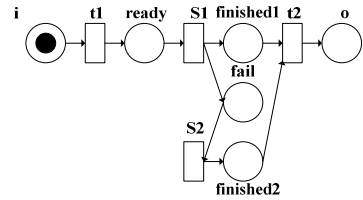

Fig. 3b Call (S1, S2)

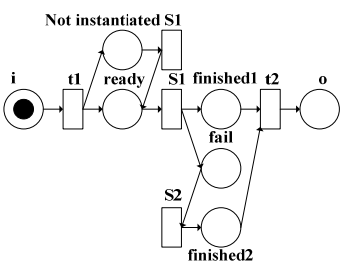

Fig. 3c Call (S1, S1and S2)

Call (S1, S1) shows the requested state of S1 is not instantiated, S1 would be dynamic extended to complete the service, as shown in Fig. 3a; Call (S1, S2) shows the requested state of S1 is fail, then call S2 to complete the service, as shown in Fig. 3b; Call (S1, S1 andS2) is formed by combining Call (S1, S2) with Call (S1, S2), as shown in Fig. 3c.

\section{Petri net-based Dynamic Extensible Service model}

Definition2. Dynamic Extensible Service: The event of dynamic extensible service is formed, when the user requests service. According to the rule, the system would search the related service in the USE (Under Service Extensible) or TS (Third-party Service), and extend service to the SP, then register and offer to the user. The flag of EF would become true. This process is named dynamic extension service ${ }^{[4]}$.

Based on the above 3 kinds of basic service model, a dynamic extensible service model is proposed, as shown in Fig. 4.
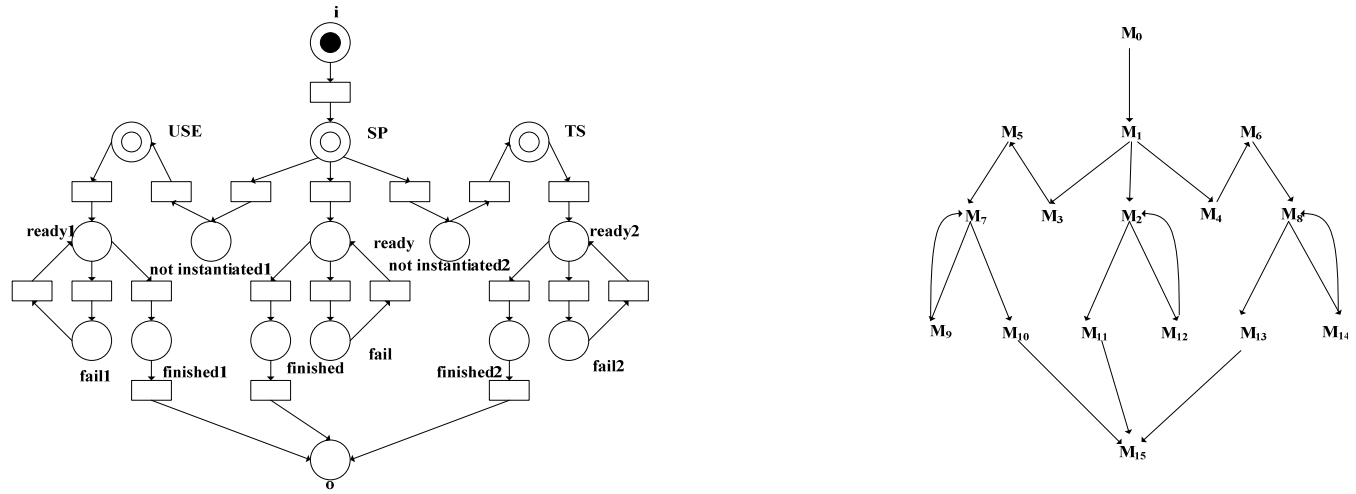

Fig.4 dynamic extensible model Fig. 5 the reachability tree of dynamic extensible model According to the user's requests, the system would search the related service in the application SP:

a. If the SP has service, the stated of service is marked with ready.

b. If not, the system will look at the service form the USE and TS:

(a) Only USE has requested services. The services are extended and the stated of service is marked with ready

(b). Only TS has requested services. The services are extended and the stated of service is marked with ready.

(c). Both of they have requested services. The services form the USE are extended and the stated of service is marked with ready.

c. When the service is unavailable during using, that is to say, the stated of service is fail. In order to ensure continuous service, the system will dynamically invoke another service from the queue of ready services. In Fig. 4 only shows the fail of services to return the queue of ready services. 
A model is correct, if and only if the Petri net which is constructed by model must have reachability, boundedness, liveness, integrity and progressivity feature. Their definitions and proof processes can be found form [5].

The analysis method of Petri nets has quit a few kinds, such as the reachability tree, reduction, invariant analysis, etc. The reachability tree is chosen in the paper. Because the analysis methods of reachability tree make the result became simple and intuitive. In addition, it is very convenient that the reachability tree is used to analyze the dynamic feature of system reachability, boundednes, liveness, etc.

\section{Correctness Analysis}

The algorithm of reachability tree is obtained from [3]. Moreover, the corresponding reachability tree is constructed and shown in Fig. 5:

Marking $\mathrm{M}_{\mathrm{i}}=$ (i, SP, ready, not instantiated1, not instantiated2, USE, TS, ready1, ready2, fail1, finished1, finished, fail, finished2, fail2, o)

In light of the reachability tree, the dynamic extensible service model is analyzed as follows:

(a) What can be seen from the reachability tree is that all the transition $t \in \mathrm{T}$ can be reach to all marking $M^{\prime} \in\left[\mathrm{M}_{0}\right.$ form $\mathrm{M}_{0}$. There is no infinite loop, and each trigger will gradually push the termination marking $\mathrm{M}_{15}$. In other words, the model is alive, reachable, integrated and progress.

(b) In the reachability tree, each state's token number is not more than 1 , that is to say $\mathrm{k}=1$ and the model is bounded and safe.

From the above analysis results, the conclusion is drawn that the model is correct. Furthermore, the analysis method of model based on Petri net is an effectiveness method.

\section{Conclusion}

In this paper, we describe and analyze the dynamic extensible model by using Petri net. The basic model of on-demand application customization service is given, which based on Petri net. Then, we show the dynamic extensible service model on the basic model. The correctness of the service dynamic extensible model is analyzed by structuring corresponding reachability tree. Based on this analysis, we demonstrate that the reachability tree of the model satisfy the demands of reachability, boundedness, liveness, progressivity and integrity. Finally, we prove that our dynamic extensible service model is correct. And it demonstrates Petri net is an effective tool to validate on-demand service dynamic extensible model.

The next step is perfecting the on-demand dynamic extensible model and how to use based on colored Petri net to describe the service model of the cloud computing.

\section{References}

[1] Foster I et al. The Grid2: Blueprint for New Computing Infrastructure. 2nd Edition. New York, USA: Morgan Kaufmann Publishers, 2004

[2] Foster I, Kesselman C, Nick J , Tucker S. T he anatomy of the Grid: Enabling scalable virtual organizations. International Journal of Supercomputer Applications, 2001, 15( 3) :200- 222

[3] Yuan Chong-yi, Petri net theory, Beijing: Electronics Industry Press, 2005.

[4] Fan Cheng, Jiang Jing, Dai Hong, Pan Zhen. Research of Service Dynamic-Extension Mechanism Based on SOA, Telecommunications Science, 2012.9

[5] WU Zhe-Hui. Petri net introduction, Beijing: Machinery Industry Press, 2006.4

[6] YU Feng, LU Jun-Zhou, LI Wei, WANG Peng. A Reachability Determining Algorithm of Petri Net Based on Process Verification. Chinese Journal of Computers,2012.2

[7] Zhou Cong-Hua, JU Shi-Guang. A Petri Net Based Approach to Covert Information Flow Analysis. Chinese Journal of Computers,2012.8

[8] YU Xiao, WU Jian-Zhang, WANG Wei. Colored Petri Net-Based Service Composition Modeling and Verification. Computer Systems \& Applications.2012.9 